Fig. 20. Schematische Grundform der "totalen Embryonalanlage" des Hühnchens. stz Stammzone derselben. sz Seitenzone. W Stelle der Wolf'schen Leiste der rechten Uälfte der Embryonalanlage, als Hervorragung zu denken. pr Primitivinne, af Amnionfalte, in derselben Form die Embryonalanlage begrenzend, durch ar, die Amnionrinae (Nabelrinne), von der Embryonalanlage getrennt; v vorne, h hinten, s seitlich. 1-6 Die verschiedenen Abschnitte (Seiten) der hexagonalen Embryonalanlage, zunächst ihrer Seitenzone. $1^{\prime}-6$ ' Die verschiedenen Abschnitte der Amnionfalte; bei $v$ der vordere Fortsatz der Embryonalanlage, bei b der hintere Fortsatz derselben, $s \mathrm{f}$ die Seitenfortsätze.

\title{
XXXII.
}

\section{Veber einen Fall von vollständiger obliteration der Arteria anonyma, fast vollständiger der carotis und subclavia sinistra, complieirt mit Aneurysma der Aorta und Carcinom des 0esophagus.}

\author{
(Aus der medicinischen Abtheilung des Cölner Bürgerhospitals.) \\ Von Dr. P. Preisendörfer, Assistenzarzt.
}

(Hierzu Taf. XVII.)

Im Folgenden erlaube ich mir Mittheilung über einen auf der Riegel'schen Abtheilung jüngst zur Beobachtung gekommenen Fall zu machen, welcher sowohl nach der klinischen als nach der anatomischen Seite manches Interessante bieten dürfte.

Mitte April des Jahres 1877 stellte sich ein Mann von 45 Jahren im Krankenhause vor, der angab, dass er seit etwa 3 Monaten an allmählich intensiver gewordenen Schlingbeschwerden leide. Während flüssige Nahrung noch ziemlich gut hinabgeschluckt werden könne, blieben consistente Nahrungsmittel auf halbem Wege sitzen. Hierzu geselle sich oft Aufstossen und Erbrechen. Patient will selt dieser Zeit stark abgemagert sein. Ausserdem hatte er noch seit Beginn seines Leidens über mehr oder weniger heftigen Hasten zu klagen; sonst war er nach seiner Angabe nie krank.

Am Tage des Eintrittes ergab sich folgender objectiver Befund:

Sebr cachectisches Ausseben, blasse Haut, das Unterhautfettgewebe äusserst spärlich; Musculatur atrophisch, keine Cyanose. Am Halse ausser einer leichten Asymmetrie beider Schildknorpelplatten nichts Abnormes; auch die laryngoskopische Ontersuchung ergiebt nur Negatives, insbesondere keine Bewegungsanomalien der 
Dirchow's Archin. Bd. LXXXIII




Stimmbänder bei Phonation und Respiration. Die Athmung etwas beschleunigt (30 p. M.), indess keine Dyspnoë. Die Verbindungsstelle des Manubrium mit dem Corpus sterni bildet einen etwas vorspringenden Winkel. An den Lungen mit Ausnahme einer leichten Spitzendifferenz zu Ungunsten der recbten Seite, vorne bis zur 2. Rippe, binten bis zur Spina scapulae, keine Veränderung, die Athmungsgeräusche vesiculär, nur an der Spitze rechterseits unbestimmtes In-, bronchiales Exspirium mit sp̧ärlichem Rasseln. Ein Herzspitzenstoss ist weder sicht- noch fühlbar, dagegen fühlt man auf dem Manubrium sterni eine mit der Herzthätigkeit synchrone Erschïtterung. Das Manubrium sterni giebt einen mässig gedämpften Schall, der beiderseits bis zur Insertion der Rippen reicht. Eine absolute Herzdämpfung nicht nachweisbar, jedoch wird der Percussionsschall unterhalb der 4. Rippe links vom Sternum kürzer. Auscuitatorisch an der normalen Stelle der Herzspitze zwei dumpfe Töne, an der Tricuspidalis hört man einen dumpfen ersten Ton, dem unmittelbar ein schwaches, hauchendes Geräusch folgt, dann einen zweiten Ton. Dieses Geräusch lässt sich längs der ganzen Ausdehnung des Sternums verfolgen und hat seine grösste Intensität einen Querfinger unterbalb der Ansatzstelle des Corpus sterni; es wird dasselbe gegen die Pulmonalis zu schwächer und lässt sich an der Stelle der Aorta nicht mehr deutlich erkennen. Carotispuls beiderseits kaum fühlbar, an der Carotis hört man zwei kurze leise Töne, auf die linke lässt sich das oben erwälnte Geräusch gut verfolgen; über der rechten ist es nur im Anfangstheil kaum angedeutet. Der Radialpuls beiderseits äusserst schwach, kaum fühlbar. Die Pulscurve der Radialis, die Herr Riegel mit dem Marey'schen Sphygmographen zeichnete, stellt eine fast gerade Linie dar, an der eben noch leichte Elevationen erkennbar sind. Im Gegensatze zu dieser kaum fühlbaren Pulsation der Carotiden und Radiales ist der Puls de Femoralis sehr kräftig, rechts etwas stärker als links; jedoch besteht vollständige Syachronie der beiderseitigen Pulse. Unterleibsorgane ohne nachweisbare Veränderung; insbesondere ist keine Vergrösserung der Leber nachzuweisen. Die eingeführte Schlundsonde dringt vom Anfang dẹ Mundhöhle aus gerechnet $36 \mathrm{~cm}$. weit in den Oesophagus vor, stösst dann auf ein nicht passirbares Hinderniss. Temperatur normal; Sputa schleimig, spärlich; Harn sauer, specifisches Gewicht 1023, frei von Albumen und Zucker.

Der weitere Verlauf des Falles war in Kürze folgender:

Die oben erwähnten Symptome blielren während der näclsstfolgenden Zeit durchaus unverändert. Am 4. Mai klagte Patient über stärkere Schmerzen in der rechten unteren Thoraxhälfte, zugleich trat jetzt mässige Temperatursteigerung auf. Die Untersuchung ergab eine leichte Schalldämpfung vom rechten unteren Scapularrand abwärts bis zur Basis der Lunge mit schwachen, unbestimmten Atlumungsgeräuschen, zugleich mit fein- und mittelblasigem feuchtem Rasseln. Eine Milzvergrösserung war nicht nachweisbar. Die Temperatur hatte am 16. Mai wieder ihre Norm erreicht und auch die Dämpfung verlor sich von da ab allmäblich wieder; im Uebrigen magerte Patient immer mehr ab und betrug dessen Körpergewicht am 9. Juli nur noch 39 Kilo. Da er um der Oesophagusstenose willen nur flüssige Nahrung zu sich nehmen konnte, auch selbst diese in letzter Zeit häufig regurgitirte, so wurden ihm jetzt neben dieser täglicl Leube'sche Fleischpankreas. 
klystiere verabfolgt $(300,0 \mathrm{Hleisch}, 100,0$ Drüse $)$, von denen auf der Riegel'seben Abtheilung früher wiederholt mit sebr gutem Erfolge Anwendung gemacht worden war. Diese Klystiere behielt unser Kranker meistens vom Abend bis zum nächsten Morgen, manchmal selbst bis Mittag bei sich. Der Effect derselben war eln so günstiger, dass das Körpergewicht am 25. Juli bereits auf 40 Kilo gestiegen war und Patient bald während der grössten Zeit des Tages das Bett verlassen und umbergehen konnte. Am 15. September betrog das Körpergewicht bereits 42 Kilo. Allmählich war Patient auch wieder im Stande consistente Nahrungsmittel in bleinen Quantitäten hinabzuschlingen.

Anfangs October trat wieder stärkerer Husten auf, die Expectoration wurde immer reichlicber, die Sputa batten bald einen rein purulenten Charakter und zeigten ausserdem sehr fötiden Geruch bei saurer Reaction. Die unterste Schichte war fast vollständig reiner Eiter. Eine Dämpfung war ausser der bereits im Anfange beschriebenen rechtsseitigen Spitzendämpfung anderweitig an den Lungen nicht nachweisbar; anscultatorisch ergaben sich viele Rbonchi über beide Lungen verbreitet. Die Temperatur, die bisher normal gewesen, zeigte jetzt wieder leichte Erhöhung. Am 16. October konnte man deutlich den Geruch der Arznei im Sputum wiedererkennen; mikroskopisch fanden sich zwar Eiterzellen, Schleim, Detritus, hie und da Muskelfasern, jedoch keine sonstigen Elemente, die einen diagnostischen Anlaltspunkt gegeben hätten. Patient collabirte von da ab sehr rasch und starb an 17. October unter den Erscheisungen von Lungenödem.

Die 24 Stunden p. m. vorgenommene 0 bduction ergab Folgendes:

Hochgradig abgemagerte Leiche obne Starre, mit wenigen Todtenflecken anf dem Rücken. Es erstreckt sich die grösste Concavität des Zwerchfells rechts bis zum oberen Rande der 6., links bis zur 5. Kippe. Bei Eröffnung der Brusthöhle retrahiren sich beide Lungen fast gar nicht. Nach Abtragung des Herzbeutels sieht man über der Ursprungsstelle der Aorta eine grosse sackförmige Geschwulst, die an ihrer Oberlạ̈che Längsfalten zeigt und die von der Ursprungsstelle der Aorta an gemessen eine Lăngenausdehnung von $15 \mathrm{Cm}$. und einen grössten Querdurchmesser von $11 \mathrm{Cm}$. besitzt; der ganze Sack ist vollkommen leer, stark gefaltet; aus seinem oberen Rande entspringen rechts der Truncus anonymus, der bei einer Länge von $3 \mathrm{Cm}$. unterhalb der Theilungsstelle einen Durchmesser von 1,5 $\mathrm{Cm}$. hat und dann in die Carotis dextra, deren Durehmesser 0,7 Cm. und Subclavia dextra, deren Durchmesser $0,9 \mathrm{~cm}$. beträgt, übergeht; links die Carotis sinistra und Subclavia sinistra, deren Durchmesser 0,8 and $0,9 \mathrm{~cm}$. sind. Es wurden nun Kehlkopf und Brusteingeweide in toto herausgenommen. Dabei fand sich die Oberfläche der linken Lunge durch ein straffes Band an die 2. Bippe geheftet, ausserdem war dieselbe in ihrem hinteren Abschnitte mit der Costalpleura verwachsen. Die rechte Pleura costalis stark injicirt, an verschiedenen Stellen durch lockere Adhäsionen mit der Pulmonalpleura verwachsen. Nach Eröffnung des Oesophagus findet sich in der Höhe der Bifurcation eine grosse ulcerirte Fläche, die die ganze Oesophaguswand einnimmt. Den hinteren rechtsseitigen Abschnitt dieser Ulcerationsfläche bildet infiltrirtes Lungengewebe von missfarbenem grauschwärzlichem Aussehen, das obne scharfe Grenze in das ulcerirte und infiltrirte Oesophagusgewebe übergeht. Die grösste Länge der ulcerirten Fläche beträgt $8,5 \mathrm{Cm}$. , die grösste Breite $8 \mathrm{Cm}$. Das 
Lungengewebe ist in einer Breitenausdehnung von $6 \mathrm{Cm}$. an der Bildung der Höble betheiligt. Dieser ganze 0 esophagusabschnitt zeigt ein missfarbenes graues Aussehen, sehr unehene 0 berfläche und fühlt sich die ganze Wand besonders an den Rändern sebr derb an. Das Gewebe selbst stark verdickt, hart, auf der Schnittläche grauweiss. Larynx and Trachea ohne bemerkenswerthe Veränderungen; Herzfleisch schlaff; die Ventrikel nicht erweitert, Wandungen nicht hypertrophisch; die Klappen nicht verändert; nur an den Nodulis der Aortenklappen einige kalkige. Einlagerungen. Die ganze Aorta ascendens, sowie der Arcus stellen einen grossen aneurysmatischen Sack dar, dessen Innenwandungen sebr uneben, mit zahlreichen Kalkplatten versehen and dessen Wand besonders an der Abyangsstelle der grossen Gefässe stark verdickt ist. Von der Aorta aus lässt sich in die Anonyma eine Sonde nicht einfuhren und ist ein Lumea nirgends sichtbar. Aa der Abgangstelle der linkseitigen Carotis und Subclavia sieht man von in uen nur kleine antersteckadelkopfgrosse 0effnungen, die nur für ganz feine Sonden passirbar sind. Von oben betrachtet sieht man, dass Platten schieberartig das Lumen an der Ursprungsstelle der genannten Gefässe verlegen.

Die rechte Lunge sehr gross, die Ränder emphysematös gebläht; im Oherlappen eine kleine, etwas unregelmässige, mit fetzigem Material gefüllte Caverne, ausserdem einzelne auf der Schnittiläche vorspringende weisse Knötchen von der Grösse eines Stecknadelkopfes, sowie mehrere schiefrig indurirte Heerde. Derjenige Lungenabschnitt, der die Wand der Speiseröhre an der früher erwähnten Stelle mitbilden bilft, zeigt schiefrige Induration. $4 \mathrm{Cm}$. unterhalb der Bifurcationsstelle lässt sich ein Bronchus mittlerer Ordnung direct in das Lumen der Geschwulstmasse resp. des Oesophagus verfolgen: An den Bronchien selbst ist eine streifige Rötbung der Schleimhaut zu erkennen, letztere ist mit zähem Schleim belegt. Die linke Lunge noch ziemlich gut lufthaltig; nur im Oberlappen einige kleinere käsige Heerde, sowie eine haselnussgrosse Caverne mit schiefriger Umgebung und schmierig käsigem Inbalt; ausserdem finden sich zerstreut noch einige kleine, vorspringende, grauweisse Knötehen.

An den übrigen Organen ist nichts Bemerkenswerthes: Milz von normaler Grösse, blass, sonst ohne Veränderung. Rinde der Nieren leicht streifig. Leber von normaler Grösse, zeigt deutliche acinöse Zeichnung. Magen und Darm bieten nichts Bemerkenswerthes.

Mässiges Oedem der Pia, Basilararterie von normaler Weite, Hirnsubstanz etwas blass, sonst ohne Veränderung. Beide Radialarterien verlaufen normal und zeigen den gewöhnlichen Durchmesser.

Die Obduction ergab also ein gleichzeitiges Bestehen dreier verschiedener Affectionen: Carcinom des Oesophagus, Aneurysma der Aorta und Tuberculose. Das Zusammentreffen von Aneurysma und Carcinom dürfte wohl ein zufälliges gewesen sein. $O b$ das Vorkommen von Aneurysma und Phthise gleichfalls auch nur ein Spiel des Zufalls oder ob die Lungenphthise ein secundärer Prozess in Folge des Aneurysma war, dürfte schwerer zu entscheiden sein. 
Zuerst hat $\mathbf{S}$ tokes auf die häufige Complieation der Aneurysmen mit Lungenphthise aufmerksam gemacht, was nach ihm mehrere Forscher bestätigten (Fuller 1:20). Hanot ${ }^{1}$ ) fand dieselbe unter 42 Fällen $16 \mathrm{mal}$ und. sucht den Grund der Häufigkeit in der Compression der Pulmonalarterie, Habersohn in einer Läsion des Nervus vagus.

Abgesehen von der hier nicht weiter in Betracht kommenden Frage einer etwaigen Beziehung des Aneurysma zur Phthise, abgesehen von der Seltenheit eines derartigen Zusammentrefiens der genannten 3 Krankheitsformen dürte der vorliegende Fall sowobl nach der anatomischen Seite wegen der hochgradigen Stenosirung der 3 grossen Gefässe zu gleicher Zeit, als insbesondere auch nach der diagnostischen Seite besonderes Interesse beanspruchen.

Wenn wir die Lungenaffection ausserhalb des Bereiches unserer Betrachtung lassen, so waren die wichtigsten objectiven Befunde während des Lebens: Das hochgradig kachektische Aussehen, die leichte Dämpfung auf dem Manubrium sterni, das längs des ganzen Sternums hörbare, wenn auch schwache Geräusch, die beträchtliche Kleinheit der Pulse der oberen gegeniber denen der unteren Körperhälfte, endlich die Stenose des Oesophagus.

Das marantische Aussehen des Patienten, die Stenose des Oesophagus, der Sitz der Stenosirung in der Höhe der Bifurcationsstelle, das Alter, all' das musste wohl sofort den Gedanken an ein Carcinom des 0esophagus nahe legen, allein die übrigen Symptome, die Dämpfung auf dem Sternum, insbesondere die Kleinheit der Pulse der oberen Körperhälfte konnten durch die Annahme eines Carcinoms des Oesophagus allein eine Erklärung nicht finden.

Eher war es vielleieht möglich, durch einen mediastinalen Tumor das ganze Symptomenbild za erklären. Welcher Art diese mediastinale Neubildung sei, ob Lipom, Sarkom, Carcinom u. dergl., diese Frage kam erst an zweiter Stelle zur Beantwortung; übrigens würden die bereits oben angeführten Gründe für ein Neoplasma maligner Art gesprochen haben. Ein solcher Tumor, der seinen Sitz in der Gegend der Abgangsstelle der grossen Gefässe hätte haben müssen, diese einerseits, den Oesophagus andererseits comprimirte, konnte recht wohl Anlass zu solchen Erscheinungen geben, wie sie

1) Hanot, Virchow-Hirsch Jahresbericht. 1876. II, S. 195. 
sich in unserem Falle fanden. Allein immerhin musste es auffallend, ja fast unmöglich erscheinen, dass die genannten Organe allein durch einen solchen Prozess in Mitleidenschaft gezogen sein sollten, während andere, insbesondere die grossen Venen und Nervenstämme, der Nervus vagus ${ }^{i} u$. dergl, vollkommen verschont blieben. Auch die Kleinheit, insbesondere die Regelmässigkeit der Dämpfung und das enge Begrenztsein derselben auf das Manubrium sprach gegen einen mediastinalen Tumor.

Ausser den genannten musste noch ein anderer Prozess in das Bereich der Möglichkeit gezogen werden, nehmlich ein Aneurysma der Aorta.

Durch ein solches waren zwar die stenotischen Erscheinungen von Seite der grossen Gefässe nicht direct erklärt, allein man konnte sie möglicherweise entstanden denken durch Ablagerung fibrinöser Schichten in dem aneurysmatischen Sacke, wie in den Fällen von Kussmaul ${ }^{1}$ ) und Tarsons ${ }^{2}$ ) oder durch entzïndliche Prozesse an der Arterienwand, die selbst zu Stenosen führen konnten, wie in dem bisher fast ganz vereinzelt dastehenden Falle Riegel's ${ }^{3}$ ). Letzterer Fall war es auch, der uns bereits Anfangs an die Möglichkeit einer derartigen Complication denken liess. Aber offenbar wäre es, wie in den angeführten Fällen, ein besonderes Spiel des Zufalls gewesen, wenn die Abgangsstellen aller grossen Gefässe der oberen Körperhälfte auf einmal zu gleicher Zeit solche Veränderungen gezejgt hätten. Es waren ferner bei Annabme eines Aortenaneurysmas noch alle jene Momente zu berücksichtigen, welche gegen die mediastinalen Tumoren sprachen, insbesondere würden wohl bei einem so grossen Aneurysma, welches vorne Dämpfung verursachte, nach rückwärts den Oesophagus comprimirte, eine Betheiligung des Recurrens und weitere Druckerscheinungen kaum ausgeblieben sein. Auch gegen diese Diagnose als letzten Grund aller pathologischen Erscheinungen sprachen darum nicht unwichtige Bedenken.

Eine letzte Affection, die vielleicht jene Symptome noch zu erklären vermochte, war die sehwielige Mediastinopericar-

1) Kussmaul, Deutsche Ḱlinik 1872. 50 u. 51.

2) Tarsons, The Boston medical and surgic. journal. Jun. 20. 1872.

$\left.{ }^{3}\right)$ Riegel, Sitzungshericht des allg. ärtlichen Vereins zu Cöln. Berl. łalin. Wochenschr. 1877. No. 21. 
ditis. Schwielige Stränge als Residuen einer abgelaufenen Mediastinopericarditis konnten die Verengerung sowohl der Gefässe, als auch des Oesophagus herbejgeführt haben, auch fand die Dämpfung auf dem Manubrium, sowie das Geräusch durch Annahme einer solchen eine hinreichende Erklärung; allein es wurden gerade 2 für jene Krankheitsform besonders charakteristische Erscheinungen vermisst, nehmlich Kussmaul's paradoxer Puls (an der Femoralis war ein solcher niemals nachzuweisen), sowie die inspiratorische Anschwellung der Halsvenen.

Fasst man diese Gesichtspunkte in's Auge, so musste man, wenn man nicht mehrere Prozesse als zu gleicher Zeit bestehend annehmen wollte, von Stellung einer sicheren Diagnose absehen; aber auch bei der am meisten wahrscheinlichen Annabme des gleichzeitigen Bestehens mehrerer krankhafter Prozesse, wie insbesondere eines Oesophaguscarcinoms und Aortenaneurysmas, waren einzelne Erscheinungen, wie das völlige Fehlen der Pulse der oberen Körperhälfte noch keineswegs in befriedigender, jedenfalls nicht in sicherer Weise erklärt. Auch im weiteren Verlaufe ergaben sich zur Feststellung einer sichern Diagnose nicht weitere Anhaltspunkte; nur musste man bei dem plötzlichen Eintritt so-massenhafter Expectoration von Eiter an eine noch nicht erwähnte Möglichkeit denken, an die Möglichkeit eines Abscesses, der in die Bronchien perforirt war. Bemerkenswerth ist noch ein weiterer Punkt, dass nehmlich trotz der hochgradigen Stenosirung der grossen Gefässe weder von Seiten des Centralnervensystems irgend welche Störungen auftraten, noch dass eine stärkere Abmagerung der oberen gegenüber der unteren Körperhälfte za erkennen war oder Störungen der Motibilität oder Sensibilität.

Die 0 bduction hatte das ganze Symptomenbild in der befriedigendsten Weise aufgeklärt; es war insbesondere durch dieselbe nachgewiesen worden, dass die fast vollständjge Pulslosigkeit der Arterien der oberen Körperhälfte ihren Grund in einem sehr seltenen Phänomen, nehmlich dem gleichzeitigen Betroffensein der 3 Ostien durch arteriitische Prozesse hatte, indem durch Verdickung der Arterienwände eine vollständige Obliteration der Anonyma, eine fast vollständige der beiden anderen grossen Gefässe berbeigeführt worden war, bei vormaler Weite der Gefässe selbst sofort oberbalb ihres Ursprungs. 
Nach Krause beträgt

der Durchmesser des normalen

Trune. anonym. . . 13,5 $\mathrm{Mm}$.

Carotis dextr. . . . 9,0 -

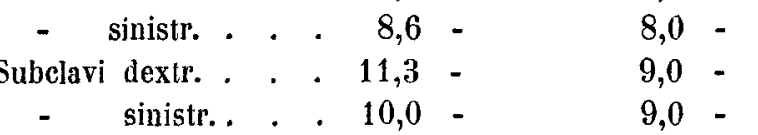

In unserem

Falle betrug derselbe

$15,0 \mathrm{Mm}$.

7,0

Eine Abbildung, die mein College Herr Dr. Tuczek mir nach dem Original zu fertigen die Güte hatte, dürfte das ganze in ausreichender Weise veranschaulichen (vergl. Tafel XVII).

Das Herz ist vom linken Ventrikel aus gegen die Aorta hin eröffnet und der aneurysmatische Sack in seiner ganzen Länge aufgeschnitten, so dass man von der gefalteten Innenfläche des Aneurysma aus erkennen kann, wie bei der linken Art. carotis und subclavia die Lumina gegen den Sack so weit verlegt sind, dass nur ganz feine Sonden sie noch zu passiren vermochten, während dieses beim Truncus anonymus nicht mehr möglich war.

Stenosen der grossen Gefässe können bekanntermaassen aus den verschiedensten Ursachen entstehen. So kommen selbst hochgradige Stenosen der Aorta und ihrer Aeste bereits als angeborene Zustände vor [vgl. Virchow ${ }^{1}$ ), Rokitansky ${ }^{2}$ )]. Diese betreffen am häufigsten die Gegend des Ductus Bolalli [rgl. die Fälle von Eppinger $\left.{ }^{3}\right)$ ]. Häufiger wird die Stenosirung durch Druck von aussen veranlasst sein oder durch Thrombenbildung im Innern. Bei einer solchen Thrombosirung der Art. subclav. dextr. konnte Heabner ${ }^{4}$ ) beobachten, wie der Puls der rechten Radialarterie immer kleiner wurde, um nach 10 Tagen vollständig zu verschwinden. In dem oben erwähnten Falle Tarsons's waren in Folge eines Traumas Schling- und Athembeschwerden eingetreten, Vergrösserung des Herzens nach rechts und systolisches Geräusch an der Mitralis und Aorta and hatte die Obduction Atherom und Erweiterung der Aorta, auf der Höhe der Aorta ein wallnussgrosses Aneurysma ergeben und waren Trunc. anonym. und Subelavia

1) Virchow, Ueber die Chlorose und die damit zusammenhängenden Anomalien im Gefässapparate. Berlin 1872.

2) Rokitansky, Pathologische Anatomie, 185b. II. S.337.

$\left.{ }^{3}\right)$ Eppinger, Prager Vierteljahrschrift Bd.112. S.31.

4) Henbuer, Archiv der Feilkunde. 1872. S. 165. 
sinistr. vollständig, Carotis sinistr. theilweise durch Fibrinpfröpfe verstopft. In dem Falle Kussmaul's war die Art. subclav. dextr. und Carot. sinistr. vollständig, die linke Subclav. theilweise durch Thromben verlegt, während die rechte Carotis noch durehgängig war. Sehr häufig sind es arterio-selerotische Prozesse, welche die Gefässlumina stenosiren; immerhin äusserst selten aber dürfte der Prozess sich an allen 3 grossen Gefässen $z u$ gleicher Zeit entwickeln, wie in dem Falle Riegel's. Herr Riegel hatte die Güte, mir die betreffende Krankengeschichte zur Verfügung zu stellen und werde ich bei der grossen Seltenheit derartiger Beobachtungen diesen bisher nur mit wenigen Worten in den Sitzungsberichten des ärztl. Vereins zu Cöln niedergelegten Fall hier anzufügen mir erlauben. Ein ähnliches Präparat von stenosirendem Atherom fand sich in unserer Sammlung pathologischer Präparate: Auch hier waren alle 3 grosșen Gefässstämme betroffen, jedoch war die Stenose nicht so hochgradig, wie in den oben erwähnten Fällen.

In dem Falle Riegel's handelte es sich um eine 49 jährige Frau. Anamnestisch bemerkenswerth war, dass seit 2 Jahren die Menses, die bis dahin immer regelmässig waren, in unregelmässigen Intervallen von 2-10 Wochen auftraten und dass sie seit dieser Zeit über starken Husten, Auswurf und Athemnoth zu klagen hatte.

Bej der Aufnahme am 11. April 1876 bot sie folgendes Bild:

Kräftige Musculatur, blasses, cyanotisches Aussehen, Jugularvenen dilatirt; überall voller Lungenschall mit vesiculären Athmungsgeräuschen, nur in den rechten hinteren Partien an der Basis dichte Bhonchi. Absolute Herzdämpfung im Längsdurchmesser normal, im Querdurchmesser vergrössert; die rechte Grenze der absoluten Herzdämpfung ist an der Mitte des Sternums. An der Mitralis hört man ein systolisches Geräusch, desgleichen ein sehr lautes an der Tricuspidalis; Aortentöne dumpf, zweiter Pulmonalton verstärkt. Ka u Carotis. und Radialpuls. Sehr stark aufgetriebener Unterleib. Unterer Leberrand in der Nabelhöhle fühlbar, derselbe fühlt sich glatt, aber derb an. Keine Milzvergrösserung, Spur von 0edem um die Knöchel. Im Harn nichts Abnormes; keine Temperaturerhöhung.

Während des Aufenthaltes im Hospitale steigerte sich die Dyspnoë häufig zu förmlicher Orthopnoë; die Diurese war stets gering. Am 11. April liess sich links hinten vom Scapularrand bis zur Basis leichte Dämpfung mit unbestimmten, schwachen Athmungsgeräuschen nachweisen, gleichzeitig mit leichter Temperaturerhöhung; das Sputum zeigte eine leicht sanguinolente, dunkelrothe Beschaffenheit. Am 18. April war der Puls an den Arterien der oberen Körperhälfte nicht mebr zu palpiren, an der Cruralis war derselbe sehr klein, $120 \mathrm{p}$. m. Beständig tiefer Sopor und 
Stöhnen. Am 20. waren die Geräusche am Herzen nicht mehr zu hören; am 22. liess sich auch rechts hinten ein kleiner Dämpfungsheerd nachweisen, das 0edem an deu Unterschenkeln nahm $z \mathbf{u}$, erstreckte sich allmäblich auch auf die Bauchdecken and so erlag die Kranke bereits nach kurzer Zeit.

Die Section 2 Tage darauf ergab:

Mässig kräftige Leiche mit Oedem der Unterschenkel und Füsse, deutliche Venenstränge am Halse, bei Eröffnung der Brustböhle sieht man von der linken Lunge nichts, sondern liegt der Herzbeutel in grosser Ausdehnung vor; im Herzbeutel wenig blutig imbibirtes Serum, etwa $\frac{1}{2}$ Liter ähnlicher Flüsslgkeit in den Pleurahöhlen; Herz scbon stark in Fäulniss übergegangen, schwach contrahirt, enthält viel Blutcoagula und Speckhaut. Klappen überall gut durchgängig, blutig imhibirt, sonst ohne Veränderung, nur die Aortenklappen an den Schliessungsrändern lejcht verdickt. Rechter Vorhof stark ausgedehnt, in der Aorta, die von normaler Weite ist, finden sich verschiedene kleinere mit Kalkplatten versehene Stellen. Direct am Anfang des Truncus anonymus ist das Lumen des Gefässes bedeutend verengt, während gleichzeitig die Gefässwand verdickt ist. Die Intima daselbst glatt und prominirt in Form einer Falte; grössere atheromatöse Stellen daselbst nicht zu erkennen. Der Anfang der rechten Arter. subclav. und carotis etwas dilatirt, die Arter. carotis und subclav. sinistr. sind an ihrem Ursprung aus dem Aortenbogen so sebr verengt, dass sich nur eine ganz dünne Borste in sie einführen lässt. Arteria radialis beiderseits ron normaler Weite.

Was die übrigen Organe betriff, so fand sich ausser mehreren Infarcten in der Lunge eine rechtsseitige kleine Ovarialcyste, Befunde chronischer Metritis und Muskatnussleber.

\title{
XXXIII.
}

\section{Experimentelle Untersuchungen über den Einfluss einiger Arsenverbindungen auf den thierischen 0rganismus.}

\author{
Von Dr. A. Lesser, \\ Assistenten an dem Institut für Staatsarzneikrnde zn Berlin.
}

(Fortsetzung von S. 408.)

\section{Rinwirknng des Arseuiks anf die Cirenlationsorgane der Homoiothermen.}

\author{
(Hierzu Taf. XVII - XIX.)
}

Eine schwache alkalische Lösung von arscnigsaurem Natron wurde den Thieren theils durch Injection in das centrale Ende der 